\title{
Metabolic effects of polycystic ovary syndrome in adolescents
}

\author{
Yejin Han, MD', \\ Hae Soon Kim, MD, PhD', \\ Hye-Jin Lee, MD, $\mathrm{PhD}^{2}$, \\ Jee-Young Oh, MD, PhD', \\ Yeon-Ah Sung, MD, $\mathrm{PhD}^{2}$ \\ Departments of ${ }^{1}$ Pediatrics and \\ ${ }^{2}$ Internal Medicine, Ewha Womans \\ University School of Medicine, Seoul, \\ Korea
}

Purpose: Polycystic ovary syndrome (PCOS) is characterized by hyperandrogenic anovulation in women of reproductive age. We investigated the metabolic effects of lean and overweight adolescents with PCOS.

Methods: Anthropometric measurements and biochemical parameters were evaluated in 49 adolescents with PCOS and 40 age- and body mass index (BMI)matched controls. We further divided both PCOS and control groups into those having $\mathrm{BMI}$ within the normal range of less than $85^{\text {th }}$ percentile and those being overweight and obese with a BMI greater than $85^{\text {th }}$ percentile.

Results: Hemoglobin, gamma-glutamyl transferase ( $r-G T)$, total cholesterol, lowdensity lipoprotein-cholesterol and 2-hour postglucose load plasma insulin levels were significantly elevated in the lean PCOS group than in the lean control group. In the overweight/obese PCOS group, hemoglobin and r-GT levels were significantly elevated than in the overweight/obese control group. In the normal weight group, none of the subjects had metabolic syndrome according to the Adult Treatment Panel III criteria, but the incidence of metabolic syndrome in the overweight/obese PCOS group was $8.3 \%$ and that in the overweight/obese control group was $6.7 \%$. Conclusion: PCOS in adolescents causes metabolic abnormalities, underscoring the importance of early diagnosis of PCOS in oligomenorrheic adolescents.

Keywords: Polycystic ovary syndrome, Metabolic syndrome, Insulin resistance
Received: 7 July, 2015

Revised: 19 August, 2015

Accepted: 21 September, 2015

Address for correspondence:

Hae Soon Kim, MD, PhD

Department of Pediatrics, Ewha

Womans University Mokdong

Hospital, Ewha Womans University

School of Medicine, 1071

Anyangcheon-ro, Yangcheon-gu,

Seoul 07985, Korea

Tel: $+82-2-2650-5569$

Fax: +82-2-2653-3718

E-mail: hyesk@ewha.ac.kr

\section{Introduction}

Polycystic ovarian syndrome (PCOS) is one of the common endocrine disorders amongst women of reproductive age, with a prevalence of $5 \%-10 \%{ }^{1)}$. The prevalence of PCOS in college students from Seoul, Korea was $4.9 \%{ }^{2)}$. PCOS is diagnosed in women in whom 2 years have passed since menarche and if she meets the criteria of the 1990 National Institute of Child Health and Human Development (NICHD) conference guideline for hyperandrogenemia with oligo-ovulation (less than 9 menstrual cycles per year) and exclusion of other known disorders such as congenital adrenal hyperplasia or Cushing's syndrome ${ }^{3)}$. The 2003 Rotterdam European Society for Human Reproduction and Embryology diagnostic criteria of PCOS consist of the occurrence of two of the following three features: (1) chronic anovulation, (2) clinical or biochemical signs of hyperandrogenism, and (3) ultrasound results indicating polycystic ovaries ${ }^{4)}$.

Insulin resistance, elevated luteinizing hormone-follicle-stimulating hormone ratio, abdominal obesity, and infertility are also common in women diagnosed with PCOS. Insulin resistance plays a key role in the occurrence of metabolic syndrome, and hyperandrogenemia is an important risk factor for metabolic syndrome in PCOS patients ${ }^{5}$. Glueck et al. ${ }^{6)}$ reported that $46 \%$ of the women with PCOS had metabolic syndrome. PCOS shares many clinical features with metabolic syndrome, including insulin resistance, obesity, type 2 diabetes mellitus (DM), hyperlipidemia, and hypertension. Among these characteristics, obesity is one of the most 
common features in women with PCOS. Approximately 50\% of women with PCOS are overweight or obese $\mathrm{F}^{7}$. Obesity is thought to be an independent risk factor of impaired glucose tolerance or type $2 \mathrm{DM}^{8)}$. However, Manco et al. ${ }^{9)}$ reported that women with PCOS had high levels of insulin resistance in both normal and overweight/obese PCOS patients. There are few studies on the metabolic abnormalities in adolescents with PCOS.

The aim of our study was to investigate metabolic abnormalities among adolescents with PCOS.

\section{Materials and methods}

The volunteers included healthy adolescents, who were high school students, having irregular menstruation and were recruited via a survey between December 2008 and October 2010. This study was performed at the Endocrinology and Gynecology Clinics at Ewha Womans University Hospital in Seoul, Korea. A trained nurse contacted eligible candidates by telephone to determine whether they were both capable and willing to participate in the study. After excluding those who did not meet the eligibility criteria, we invited volunteers to visit our hospital in the morning of the third day of their menstrual period following an overnight fast for at least 8 hours. We conducted physical examinations of 49 PCOS students and 40 control students between the ages of 16 and 18 years.

Students were diagnosed with PCOS if it had been 2 years since their menarche and if they met the criteria of the 1990 NICHD conference guideline, i.e., hyperandrogenemia with oligo-ovulation (less than 9 menstrual cycles per year), and exclusion of other known disorders, such as congenital adrenal hyperplasia or Cushing's syndrome. Women with abnormal thyroid-stimulating hormone $(<0.3$ or $>5.0 \mathrm{mIU} /$ $\mathrm{L})$ or prolactin levels $(>25 \mu \mathrm{g} / \mathrm{L})$ were excluded. Biochemical hyperandrogenemia is defined as a total testosterone level above the 95th percentile (total testosterone $\geq 67 \mathrm{ng} / \mathrm{dL}$ ) based on the testosterone levels in 1,120 healthy women with regular menstrual cycles ${ }^{10)}$. Clinical hyperandrogenism is defined as hirsutism with a modified Ferriman-Gallwey (mFG) score ${ }^{11}$.

After overnight fasting at least for 8 hours, fasting blood test, hormonal profiling and ovarian ultrasonography were performed in the early follicular phase. Testosterone levels were measured by a chemiluminescent immunoassay using a commercial kit (Siemens, New York, NY, USA). Fasting plasma glucose (FPG) level was analyzed using the glucose oxidase method (Beckman Instruments, Fullerton, CA, USA), and 2-hour postglucose load plasma glucose (2h-PG) levels were obtained using a standard 75-g oral glucose tolerance test. Fasting plasma insulin (FPI) and 2-hour postglucose load plasma insulin (2h-PI) concentrations were measured by radioimmunoassay using a commercial kit (Diagnostic Products Corp., Los Angeles, CA, USA).

Anthropometric measurements and body composition analyses were performed, and both systolic and diastolic blood pressures were measured twice using a sphygmomanometry, following a 10-minute rest, and mean values were recorded.
The control group consisted of students who had normal menstrual cycles as well as similar body mass indexes (BMIs) and age as the PCOS group. Then, we further divided the two groups into those having within normal BMI of less than 85th percentile for sex and age and those being overweight and obese with a BMI greater than 85th percentile for sex and age.

The diagnosis of metabolic syndrome was made when more than 3 of the following risk factors were present: waist circumference, $>80 \mathrm{~cm}$; systolic blood pressure, $130 \mathrm{mmHg}$; or diastolic blood pressure, $85 \mathrm{mmHg}$; and levels of fasting glucose, $110 \mathrm{mg} / \mathrm{dL}$; fasting triglycerides, $150 \mathrm{mg} / \mathrm{dL}$; and high-density lipoprotein (HDL)-cholesterol, $<50 \mathrm{mg} / \mathrm{dL}$, according to the National Cholesterol Education Program Adult Treatment Panel III (ATP III) criteria $^{12)}$.

This study was approved by the Institutional Review Board of Ewha Womans University, Mokdong Hospital. Informed consent was obtained from a legal guardian of each subject less than 18 years old.

Data analyses were performed using SPSS ver. 16.0 (SPSS Inc., Chicago, IL, USA). All data were expressed as the mean \pm standard deviation. All $P$-values were two-tailed, and statistical significance was defined as $P<0.05$.

\section{Results}

\section{PCOS characteristics and anthropometric measurements}

The frequency of menstruation was $5.3 \pm 2.4$ times per year in the PCOS group and $10.0 \pm 0$ times per year in the control group. The mean Ferriman-Gallwey score was $1.78 \pm 2.9$ in the PCOS group and $0.8 \pm 1.3$ in the control group. The testosterone level was significantly higher in the PCOS group than in the

Table 1. Characteristics and anthropometric measurements of the PCOS and control groups

\begin{tabular}{|c|c|c|c|}
\hline Characteristic & $\begin{array}{c}\text { PCOS } \\
(n=49)\end{array}$ & $\begin{array}{c}\text { Control } \\
(n=40)\end{array}$ & $P$-value \\
\hline Age (yr) & $17.1 \pm 0.7$ & $16.9 \pm 0.6$ & 0.150 \\
\hline $\begin{array}{l}\text { Frequency of menstruation per year } \\
\text { (time) }\end{array}$ & $5.3 \pm 2.4$ & $10.0 \pm 0$ & 0.000 \\
\hline FG score & $1.78 \pm 2.9$ & $0.8 \pm 1.3$ & 0.051 \\
\hline $\mathrm{PCOM}^{\mathrm{a})}(\%)$ & 75.5 & 0 & 0.000 \\
\hline Testosterone (ng/dL) & $76.5 \pm 1.97$ & $43.0 \pm 14.1$ & 0.002 \\
\hline Birth weight (kg) & $3.1 \pm 0.6$ & $3.0 \pm 0.4$ & 0.955 \\
\hline Age at Menarche (yr) & $12.6 \pm 1.6$ & $12.6 \pm 1.1$ & 0.860 \\
\hline Body mass index $\left(\mathrm{kg} / \mathrm{m}^{2}\right)$ & $22.9 \pm 2.5$ & $22.1 \pm 2.9$ & 0.199 \\
\hline Fat mass $(\mathrm{kg})$ & $20.8 \pm 5.5$ & $20.3 \pm 6.2$ & 0.701 \\
\hline Fat $\%$ & $34.6 \pm 5.5$ & $34.5 \pm 6.0$ & 0.929 \\
\hline
\end{tabular}

Values are presented as mean \pm standard deviation unless otherwise indicated.

PCOS, polycystic ovary syndrome; FG score, Ferriman-Gallwey score.

a)PCOM, PCOS morphology: ultrasound results indicating polycystic ovaries according to the European Society for Human Reproduction and Embryology consensus 
control group. The incidence rate of polycystic ovary in the PCOS group was $75.5 \%$. There was no significant difference in birth weight, age at menarche, BMI, fat mass, and fat percent between the PCOS and control groups (Table 1). There were no significant differences in weight, height, BMI, waist circumference, systolic and diastolic blood pressures, fat mass, and fat percentage between PCOS and control groups in each of their corresponding lean, overweight/obese groups (Table 2).

\section{Comparison of laboratory findings between PCOS and control in lean and overweight/obese group}

Levels of hemoglobin, total cholesterol, low-density lipoprotein (LDL)-cholesterol, gamma-glutamyl transferase (r-GT), and 2h-PI were significantly elevated in the lean PCOS group than in the lean control group. FPG, FPI, 2h-PG, fasting glucose/insulin ratio and homeostatic model assessment-insulin resistance were not significantly different between the lean PCOS and control groups (Fig. 1). As for the overweight/obese PCOS group, hemoglobin and r-GT levels were significantly elevated in the PCOS group compared to those in the overweight/obese control group (Table 3).

According to the ATP III criteria, there were no cases of metabolic syndrome in the lean PCOS and lean control groups. In the overweight/obese group, the incidence rates of metabolic syndrome in the PCOS and control groups were $8.3 \%$ and $6.7 \%$, respectively, and there was no significant difference between the two groups (Table 4).

\section{Discussion}

PCOS is a complex metabolic disorder and it is increasingly recognized in the young women. Women diagnosed with PCOS have significant insulin resistance, compensatory hyperinsulinemia, impaired glucose tolerance, dyslipidemia, and metabolic syndrome ${ }^{13-14}$. They also have elevated insulin resistance compared to BMI-matched controls. Grulet et al. ${ }^{15)}$ reported that PCOS is associated with a significant decrease of insulin sensitivity and was an independent factor of obesity. In our adolescent study, 2h-PI level was significantly elevated in the lean PCOS group compared to the lean control group. Therefore, PCOS can be an independent risk factor for insulin resistance regardless of obesity. Manco et al. ${ }^{9)}$ reported that PCOS patients with normal glucose tolerance are more insulin resistant than controls, and their insulin secretion increases to maintain normal glucose level. Our study showed similar results in that our lean PCOS group was not significantly different in the fasting glucose level and 2h-PG level compared to the lean control group. However, in the lean PCOS group, 2h-PI level was higher than that of the lean control group.

Insulin resistance appears to be related to excessive serine phosphorylation of the insulin receptor, and it is thought to modulate the activity of the key regulatory enzyme of androgen biosynthesis, $\mathrm{P} 450 \mathrm{c} 17^{16)}$. Wild et al. ${ }^{17)}$ reported that dyslipidemia is probably secondary to insulin resistance because insulin is a major regulator of cholesterol production, and hyperandrogenism may affect lipoproteins and lipids independently of insulin levels and body weight. Coviello et al. ${ }^{5)}$

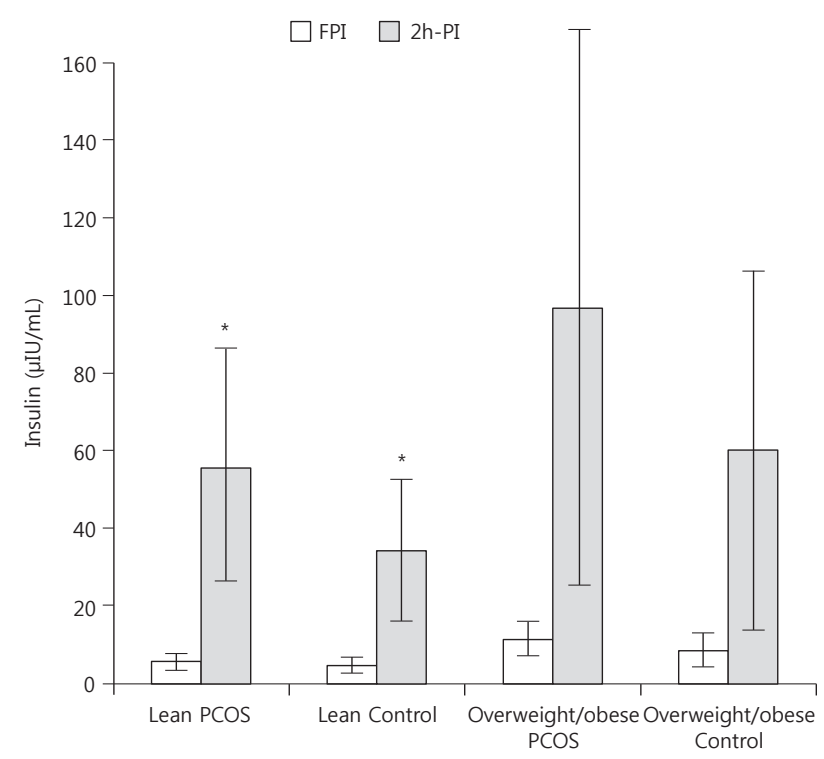

Fig. 1. Comparison of fasting plasma insulin (FPI) and 2-hour postglucose load plasma insulin (2h-PI). 2h-PI was significantly elevated in the lean polycystic ovary syndrome (PCOS) group than in the lean control group. ${ }^{*} P<0.05$.

Table 2. Comparison of anthropometric measurements among PCOS patients and controls in the lean and overweight/obese group

\begin{tabular}{|c|c|c|c|c|c|c|}
\hline \multirow{2}{*}{ Variable } & \multicolumn{3}{|c|}{ Lean group } & \multicolumn{3}{|c|}{ Overweight/obese group } \\
\hline & $P \operatorname{COS}(n=25)$ & Control $(n=25)$ & $P$-value & $P \operatorname{COS}(n=24)$ & Control $(n=15)$ & $P$-value \\
\hline Weight (kg) & $53.4 \pm 4.6$ & $53.2 \pm 6.0$ & 0.890 & $65.5 \pm 6.3$ & $65.4 \pm 8.6$ & 0.945 \\
\hline Height $(\mathrm{cm})$ & $160.2 \pm 4.6$ & $161.6 \pm 4.9$ & 0.322 & $161.5 \pm 4.8$ & $161.4 \pm 5.76$ & 0.352 \\
\hline Body mass index $\left(\mathrm{kg} / \mathrm{m}^{2}\right)$ & $20.8 \pm 1.1$ & $20.4 \pm 1.9$ & 0.325 & $25.1 \pm 1.5$ & $25.1 \pm 1.9$ & 0.962 \\
\hline Waist circumference (cm) & $70.8 \pm 3.9$ & $70.8 \pm 6.5$ & 1.000 & $81.8 \pm 5.5$ & $82.3 \pm 7.6$ & 0.802 \\
\hline $\mathrm{SBP}(\mathrm{mmHg})$ & $108.4 \pm 8.8$ & $106.8 \pm 6.7$ & 0.437 & $109.5 \pm 10.1$ & $115.3 \pm 8.1$ & 0.070 \\
\hline $\mathrm{DBP}(\mathrm{mmHg})$ & $69.8 \pm 3.2$ & $67.6 \pm 6.1$ & 0.266 & $81.8 \pm 5.5$ & $82.3 \pm 8.6$ & 0.210 \\
\hline Fat mass (kg) & $16.8 \pm 3.2$ & $16.8 \pm 3.9$ & 0.981 & $25.0 \pm 4.2$ & $26.2 \pm 4.5$ & 0.387 \\
\hline Fat $\%$ & $31.4 \pm 4.4$ & $31.2 \pm 4.6$ & 0.913 & $37.9 \pm 4.3$ & $39.9 \pm 3.8$ & 0.159 \\
\hline
\end{tabular}

Values are presented as mean \pm standard deviation.

PCOS, polycystic ovary syndrome; SBP, systolic blood pressure; DBP, diastolic blood pressure. 
Table 3. Laboratory findings of women with PCOS and controls in the lean and overweight/obese group

\begin{tabular}{|c|c|c|c|c|c|c|}
\hline \multirow{2}{*}{ Variable } & \multicolumn{3}{|c|}{ Lean group } & \multicolumn{3}{|c|}{ Overweight/obese group } \\
\hline & PCOS & Control & $P$-value & PCOS & Control & $P$-value \\
\hline $\mathrm{Hb}(\mathrm{g} / \mathrm{dL})$ & $13.4 \pm 0.7$ & $12.1 \pm 0.9$ & 0.031 & $13.0 \pm 0.9$ & $12.4 \pm 0.9$ & 0.042 \\
\hline AST (IU/L) & $20.4 \pm 5.0$ & $18.7 \pm 3.4$ & 0.164 & $20.3 \pm 2.7$ & $20.5 \pm 3.8$ & 0.868 \\
\hline ALT (IU/L) & $16.0 \pm 9.9$ & $13.6 \pm 3.7$ & 0.249 & $20.5 \pm 9.8$ & $18.2 \pm 7.1$ & 0.437 \\
\hline $\mathrm{TC}(\mathrm{mg} / \mathrm{dL})$ & $177.6 \pm 23.9$ & $162.2 \pm 25.1$ & 0.032 & $174.7 \pm 23.0$ & $168.3 \pm 20.6$ & 0.382 \\
\hline $\mathrm{TG}(\mathrm{mg} / \mathrm{dL})$ & $87.9 \pm 33.5$ & $80.0 \pm 34.2$ & 0.458 & $104.7 \pm 51.9$ & $111.9 \pm 57.7$ & 0.688 \\
\hline $\mathrm{HDL}(\mathrm{mg} / \mathrm{dL})$ & $51.3 \pm 10.0$ & $50.6 \pm 12.9$ & 0.817 & $47.1 \pm 10.8$ & $43.1 \pm 9.2$ & 0.242 \\
\hline LDL (mg/dL) & $108.7 \pm 19.2$ & $95.5 \pm 19.8$ & 0.021 & $106.7 \pm 18.7$ & $102.8 \pm 17.4$ & 0.520 \\
\hline$r-G T(I U / L)$ & $12.1 \pm 1.9$ & $9.15 \pm 2.6$ & 0.001 & $14.1 \pm 6.7$ & $9.7 \pm 3.2$ & 0.024 \\
\hline $\mathrm{FPG}(\mathrm{mg} / \mathrm{dL})$ & $86.9 \pm 8.1$ & $88.3 \pm 7.3$ & 0.670 & $86.4 \pm 7.1$ & $85.2 \pm 7.4$ & 0.622 \\
\hline $2 \mathrm{~h}-\mathrm{PG}(\mathrm{mg} / \mathrm{dL})$ & $102.3 \pm 18.9$ & $94.0 \pm 16.5$ & 0.106 & $108.5 \pm 21.5$ & $98.6 \pm 22.2$ & 0.174 \\
\hline $\mathrm{FPI}(\mu \mathrm{IU} / \mathrm{mL})$ & $5.6 \pm 3.5$ & $4.8 \pm 3.3$ & 0.511 & $11.4 \pm 7.2$ & $8.6 \pm 6.3$ & 0.324 \\
\hline 2h-PI ( $\mu \mathrm{IU} / \mathrm{mL})$ & $55.6 \pm 32.3$ & $34.2 \pm 18.1$ & 0.022 & $96.8 \pm 71.0$ & $60.2 \pm 46.9$ & 0.166 \\
\hline Fasting GIR & $21.7 \pm 22.1$ & $31.9 \pm 49.1$ & 0.397 & $11.1 \pm 7.7$ & $20.4 \pm 26.3$ & 0.314 \\
\hline HOMA-IR & $1.2 \pm 0.7$ & $1.13 \pm 0.6$ & 0.558 & $2.45 \pm 1.6$ & $1.83 \pm 1.32$ & 0.205 \\
\hline
\end{tabular}

Values are presented as mean \pm standard deviation.

PCOS, polycystic ovary syndrome; Hb, hemoglobin; AST, aspartate aminotransferase; ALT, alanine transaminase; TC, total cholesterol; TG, triglyceride; HDL, high-density lipoprotein cholesterol; LDL, low-density lipoprotein cholesterol; r-GT, gamma-glutamyl transpeptidase; FPG, fasting plasma glucose; 2h-PG, 2-hour post glucose load plasma glucose; FPl, fasting plasma insulin; 2h-PI, 2-hour postglucose load plasma insulin; Fasting GIR, fasting glucose insulin ratio; HOMA-IR, homeostasis model assessment index for insulin resistance.

Table 4. The number of metabolic syndrome component according to the ATP III criteria

\begin{tabular}{|c|c|c|c|c|}
\hline \multirow[b]{2}{*}{ Number } & \multicolumn{2}{|c|}{ Lean group } & \multicolumn{2}{|c|}{ Overweight/obese group } \\
\hline & $\begin{array}{l}\text { PCOS } \\
(n=25)\end{array}$ & $\begin{array}{l}\text { Control } \\
(n=25)\end{array}$ & $\begin{array}{c}P C O S \\
(n=24)\end{array}$ & $\begin{array}{l}\text { Control } \\
(n=15)\end{array}$ \\
\hline 0 & $13(52.0)$ & $16(64.0)$ & $14(58.3)$ & $4(26.7)$ \\
\hline 1 & $7(28.0)$ & $6(24.0)$ & $4(16.7)$ & $6(40.0)$ \\
\hline 2 & $5(20.0)$ & $3(12.0)$ & $4(16.7)$ & $4(26.7)$ \\
\hline 3 & $0(0)$ & $0(0)$ & $1(4.2)$ & $1(6.7)$ \\
\hline 4 & $0(0)$ & $0(0)$ & $1(4.2)$ & $0(0)$ \\
\hline$M S^{a)}$ & $0(0)$ & $0(0)$ & $2(8.3)$ & $1(6.7)$ \\
\hline
\end{tabular}

Values are presented as number (\%).

PCOS, polycystic ovary syndrome; ATP, Adult Treatment Panel.

a) MS: metabolic syndrome, defined as the presence of at least three of the ATP III criteria.

reported that hyperandrogenemia is an important risk factor for metabolic syndrome in women with PCOS in addition to obesity and insulin resistance. The interaction between insulin resistance, dyslipidemia, and hyperandgrogenism in PCOS makes a vicious cycle, and highlights the timely diagnosis and treatment of PCOS ${ }^{18)}$.

Various types of dyslipidemia in adolescent PCOS have been described in many studies, and most studies have reported decreased HDL cholesterol level and increased triglyceride level ${ }^{19)}$. In our study, the lean PCOS group had significantly higher total cholesterol, LDL-cholesterol compared to lean control group. However, both HDL-cholesterol and triglyceride levels were not significantly different between the lean groups of both PCOS and control groups. Like our study, some other studies reported no difference in triglyceride levels between PCOS and weight-matched controls similar to our study findings ${ }^{20,21)}$. Dejager et al. ${ }^{20)}$ detected a negative correlation between LDL particle size and triglyceride level, and suggested that triglyceride was the major determinant of LDL particle size. In addition, other abnormalities, except those in triglyceride, may be primarily responsible for the shift towards smaller LDL particles in women with PCOS. Legro et al. ${ }^{22}$ reported that total cholesterol and LDL-cholesterol levels are significantly higher in women with PCOS than in controls, but the differences in terms of triglyceride levels were eliminated after adjustment for life style factors like smoking, age, and alcohol use. Testosterone did not have a significant effect on triglyceride levels, but circulating androgen levels and no other lipids were associated with triglyceride levels among women with $\mathrm{PCOS}^{23}$. This might have been due to the peripheral conversion of androgens to estrogen or the hyperinsulinemia-mediated-increase in estrogen levels ${ }^{24,25)}$.

r-GT levels are usually increased in patients with liver disease, and a good predictor for metabolic syndrome and cardiovascular risk. In PCOS women, r-GT in the upper range is a strong independent biomarker for the metabolic syndrome ${ }^{26)}$. In our study, the r-GT levels of PCOS groups were significantly elevated than those of the control groups, both in the lean and overweight/obese groups. Although they were in the reference range, this elevation might suggest the possibilites of metabolic derangement in the PCOS groups.

In our study, hemoglobin levels were significantly elevated in both lean and overweight/obese PCOS groups compared to those in the control groups. Many studies evaluate the hematologic effect on PCOS and positive correlations between iron status parameters and serum testosterone, prolactin, and insulin in PCOS women are reported ${ }^{27,28}$. Hormone levels in PCOS women affect hemoglobin levels. Testosterone is a hematopoietic hormone and has a dose-dependent stimulatory 
effect on erythropoiesis ${ }^{29,30)}$. And androgen affects bone marrow cells via androgen receptor in the bone marrow ${ }^{31)}$. Also, reduced frequency of menstruation in PCOS group is thought to cause the differences of hemoglobin level between PCOS and control groups. Menstruation throughout adolescent years increases the risk for iron deficiency anemia in females ${ }^{32,33)}$. Our result also shows significant differences in testosterone levels and menstruation frequency, so these differences seems to have resulted in significant elevation in hemoglobin levels in PCOS group.

This study showed that the adolescents with PCOS had significantly higher total cholesterol, LDL-cholesterol compared to those in the control group. Even though levels of fasting glucose and oral glucose loading glucose were similar, $2 \mathrm{~h}$-PI levels were differed significantly between the PCOS and control groups. Therefore, this study suggests that PCOS influences metabolic abnormalities even in adolescents. However, the metabolic derangements between PCOS and control groups in overweight/obese group were not significantly distinct. In this study, the overall prevalence of metabolic syndrome was $8.3 \%$ in overweight/obese PCOS women and $6.7 \%$ in overweight/obese control subjects. We suspected that both obesity and PCOS are associated with unfavorable lipid profiles and increased insulin resistance rendering the effects on PCOS indiscriminately ${ }^{34,35)}$. And this value was much lower than that in Korean adults with PCOS $(14 \%-18 \%)^{36,37}$. We think that PCOS is a metabolic disorder that begins to emerge since pubertal onset, so perhaps because our adolescents were in the early stages of PCOS, and the prevalence of metabolic syndrome was lower than that in adults with PCOS.

A limitation of our study is that we had small sample size. We tried to control for the BMI values that have similar effects on insulin resistance with PCOS subjects, resulting in small group sizes. Therefore, this made it hard to prove the significant difference between PCOS and control in the overweight/obese group. Further studies will be needed to include larger groups of adolescents with PCOS to measure lipid profiles and hormonal factors with controlled life style factors ${ }^{38,49}$.

Establishing a diagnosis of PCOS in adolescents is not simple because features of PCOS overlap normal pubertal physiological events. After menarche during normal puberty, acne, irregular menstruation, and PCOS morphology is common so it is not specific in adolescent with PCOS ${ }^{40}$. In this study we used National Institutes of Health criteria for the diagnosis of PCOS because adolescents without hyperandrogenemia or with normal menstrual cycle could be diagnosed as PCOS when using Rotterdam criteria. However, early detection and treatment of PCOS are important because PCOS is thought to increase metabolic and cardiovascular risks and is known to be associated with reproductive morbidity and increased risk for endometrial cancer. Therefore, pediatricians should be aware of early and precise diagnosis of PCOS in the adolescent girls who show oligomenorrheia oligomenorrhea since menarche.

In conclusion, we found that adolescent PCOS patients showed metabolic derangement, underscoring the importance of early diagnosis of PCOS in oligomenorrheic adolescents.

\section{Conflict of interest}

No potential conflict of interest relevant to this article was reported.

\section{References}

1. Knochenhauer ES, Key TJ, Kahsar-Miller M, Waggoner W, Boots LR, Azziz R. Prevalence of the polycystic ovary syndrome in unselected black and white women of the southeastern United States: a prospective study. J Clin Endocrinol Metab 1998;83:3078-82.

2. Byun EK, Kim HJ, Oh JY, Hong YS, Sung YA. The prevalence of polycystic ovary syndrome in college students from Seoul. J Korean Soc Endocrinol 2005;20:120-6.

3. Zawadzki JK, Dunaif A. Diagnostic criteria for polycystic ovary syndrome: towards a rational approach. In: Dunaif A, Givens JR, Haseltine F, Merriam GR, editors. Polycystic ovary syndrome. Boston: Blackwell Scientific Publications, 1992:377-84.

4. Rotterdam ESHRE/ASRM-Sponsored PCOS consensus workshop group. Revised 2003 consensus on diagnostic criteria and long-term health risks related to polycystic ovary syndrome (PCOS). Hum Reprod 2004; 19:41-7.

5. Coviello AD, Legro RS, Dunaif A. Adolescent girls with polycystic ovary syndrome have an increased risk of the metabolic syndrome associated with increasing androgen levels independent of obesity and insulin resistance. J Clin Endocrinol Metab 2006;91:492-7.

6. Glueck CJ, Papanna R, Wang P, Goldenberg N, Sieve-Smith $\mathrm{L}$. Incidence and treatment of metabolic syndrome in newly referred women with confirmed polycystic ovarian syndrome. Metabolism 2003;52:908-15.

7. Pasquali R, Casimirri F. The impact of obesity on hyperandrogenism and polycystic ovary syndrome in premenopausal women. Clin Endocrinol (Oxf) 1993;39:116.

8. Norman RJ, Masters L, Milner CR, Wang JX, Davies MJ. Relative risk of conversion from normoglycaemia to impaired glucose tolerance or non-insulin dependent diabetes mellitus in polycystic ovarian syndrome. Hum Reprod 2001;16:1995-8.

9. Manco M, Castagneto-Gissey L, Arrighi E, Carnicelli A, Brufani C, Luciano R, et al. Insulin dynamics in young women with polycystic ovary syndrome and normal glucose tolerance across categories of body mass index. PloS One 2014;9:e92995.

10. Vermeulen A, Verdonck L, and Kaufman JM. A critical evaluation of simple methods for the estimation of free testosterone in serum. J Clin Endocrinol Metab 1999;84:3666-72.

11. Escobar-Morreale HF, Carmina E, Dewailly D, Gambineri A, Kelestimur F, Moghetti P, et al. Epidemiology, diagnosis and management of hirsutism: a consensus statement by 
the androgen excess and polycystic ovary syndrome society. Hum Reprod Update 2012;18:146-70.

12. Expert Panel on Detection, Evaluation, and Treatment of High Blood Cholesterol in Adults. Executive summary of the third report of the National Cholesterol Education Program (NCEP) expert panel on detection, evaluation, and treatment of high blood cholesterol in adults (Adult Treatment Panel III). JAMA 2001;285:2486-97.

13. Dunaif A. Insulin action in the polycystic ovary syndrome. Endocrinol Metab Clin North Am 1999;28:341-59.

14. Silfen ME, Denburg MR, Manibo AM, Lobo RA, Jaffe R, Ferin M, et al. Early endocrine, metabolic, and sonographic characteristics of polycystic ovary syndrome (PCOS): comparison between nonobese and obese adolescents. J Clin Endocrinol Metab 2003;88:4682-8.

15. Grulet H, Hecart AC, Delemer B, Gross A, Sulmont V, Leutenegger $\mathrm{M}$, et al. Roles of $\mathrm{LH}$ and insulin resistance in lean and obese polycystic ovary syndrome. Clin Endocrinol (Oxf) 1993;38:621-6.

16. Dunaif A. Insulin Resistance and the polycystic ovary syndrome: mechanism and implications for pathogenesis. Endocr Rev 1997;18:774-800.

17. Wild RA, Painter PC, Coulson PB, Carruth KB, Ranney GB. Lipoprotein lipid concentrations and cardiovascular risk in women with polycystic ovary syndrome. J Clin Endocrinol Metab 1985;61:946-51.

18. Sozen I, Arici A. Hyperinsulinism and its interaction with hyperandrogenism in polycystic ovary syndrome. Obstet Gynecol Surv 2000;55:321-8.

19. Diamanti-Kandarakis E, Papavassiliou AG, Kandarakis SA, Chrousos GP. Pathophysiology and types of dyslipidemia in PCOS. Trends Endocrinol Metab 2007;18:280-5.

20. Dejager S, Pichard C, Giral P, Bruckert E, Federspield MC, Beucler I, et al. Smaller LDL particle size in women with polycystic ovary syndrome compared to controls. Clin Endocrinol (Oxf) 2001;54:455-62.

21. Orio F Jr, Palomba S, Spinelli L, Cascella T, Tauchmanovà L, Zullo F, et al. The cardiovascular risk of young women with polycystic ovary syndrome: an observational, analytical, prospective case-control study. J Clin Endocrinol Metab 2004;89:3696-701.

22. Legro RS, Kunselman AR, Dodson WC, Dunaif A. Prevalence and predictors of risk for type 2 diabetes mellitus and impaired glucose tolerance in polycystic ovary syndrome: a prospective, controlled study in 254 affected women. J Clin Endocrinol Metab 1999;84:165-9.

23. Arslanian SA, Lewy VD, Danadian K. Glucose intolerance in obese adolescents with polycystic ovary syndrome: roles of insulin resistance and $\beta$-cell dysfunction and risk of cardiovascular disease. J Clin Endocrinol Metab 2001;86:66-71.

24. Edman CD, MacDonald PC. Effect of obesity on coriversion of plasma androstenedione to estrone in ovulatory and anovulatory young women. Am J Obstet Gynecol 1978;130:456-61.
25. Nestler JE, Powers LP, Matt DW, Steingold KA, Plymate SR, Rittmaster RS, et al. A direct effect of hyperinsulinemia on serum sex hormone-binding globulin levels in obese women with the polycystic ovary syndrome. J Clin Endocrinol Metab 1991;72:83-9.

26. Economou F, Xyrafis X, Livadas S, Androulakis II, Argyrakopoulou G, Christakou CD, et al. In overweight/obese but not in normal-weight women, polycystic ovary syndrome is associated with elevated liver enzymes compared to controls. Hormones (Athens) 2009;8:199-206.

27. Al-Hakeim HK. Correlation Between Iron Status Parameters and Hormone Levels in Women with Polycystic Ovary Syndrome. Clin Med Insights Womens Health 2012;5:1-8.

28. Supriya P, Bitla A, Prasad NR, Rajagopal G, Rao AM, Suchitra M, et al. Body iron status and association with hyperinsulinaemia and hyperandrogenism in non-obese Indian women with polycystic ovarian syndrome. J Clin Sci Res 2012;1:2-7.

29. Berria R, Gastaldelli A, Lucidi S, Belfort R, De Filippis E, Easton C, et al. Reduction in hematocrit level after pioglitazone treatment is correlated with decreased plasma free testosterone level, not hemodilution, in women with polycystic ovary syndrome. Clin Pharmacol Ther 2006;80:105-14.

30. Coviello AD, Kaplan B, Lakshman KM, Chen T, Singh $\mathrm{AB}$, Bhasin $\mathrm{S}$. Effects of graded doses of testosterone on erythropoiesis in healthy young and older men. J Clin Endocrinol Metab 2008;93:914-9.

31. Claustres M, Sultan C. Androgen and erythropoiesis: evidence for an androgen receptor in erythroblasts from human bone marrow cultures. Horm Res 1988;29:17-22.

32. Yip R, Parvanta I, Cogswell ME, McDonnell SM, Bowman BA, Grummer-Strawn LM, et al. Recommendations to prevent and control iron deficiency in the United States. MMWR Recomm Rep 1998:i-29.

33. Bitzer J, Sultan C, Creatsas G, Palacios S. Gynecological care in young women: a high-risk period of life. Gynecol Endocrinol 2014;30:542-8.

34. Friedland O, Nemet D, Gorodnitsky N, Wolach B, Eliakim A. Obesity and lipid profiles in children and adolescents. J Pediatr Endocrinol Metab 2002;15:1011-6.

35. Steinberger J, Moorehead C, Katch V, Rocchini AP. Relationship between insulin resistance and abnormal lipid profile in obese adolescents. J Pediatr 1995;126:690-5.

36. Park HR, Choi Y, Lee HJ, Oh JY, Hong YS, Sung YA. The metabolic syndrome in young Korean women with polycystic ovary syndrome. Diabetes Res Clin Pract 2007;77 Suppl 1:S243-6.

37. Chae SJ, Kim JJ, Choi YM, Hwang KR, Jee BC, Ku SY, et al. Clinical and biochemical characteristics of polycystic ovary syndrome in Korean women. Hum Reprod 2008;23:192431.

38. Lobo RA, Carmina E. The importance of diagnosing the polycystic ovary syndrome. Ann Intern Med 2000;132:98993. 
39. Macut D, Micic D, Cvijovic G, Sumarac M, Kendereski A, Zoric S, et al. Cardiovascular risk in adolescent and young adult obese females with polycystic ovary syndrome (PCOS). J Pediatr Endocrinol Metab 2001;14 Suppl 5:135359 .
40. Legro RS, Arslanian SA, Ehrmann DA, Hoeger KM, Murad $\mathrm{MH}$, Pasquali R, et al. Diagnosis and treatment of polycystic ovary syndrome: an Endocrine Society clinical practice guideline. J Clin Endocrinol Metab 2013;98:4565-92. 